\title{
In vivo magnetic resonance imaging of orthotopic prostate cancer
}

Murali K Ravoori ${ }^{1}$, Sheela Singh ${ }^{1}$, Peiying Yang ${ }^{2}$, Wei Wei ${ }^{3}$, Huiqin Chen ${ }^{3}$, Jingfei Ma ${ }^{4}$, James A Bankson ${ }^{4}$ \& Vikas Kundra*, ${ }^{1,5}$

${ }^{1}$ Department of Cancer Systems Imaging, The University of Texas MD Anderson Cancer Center, 1400 Pressler St, Houston, TX 77030 , USA; ${ }^{2}$ Department of General Oncology, The University of Texas MD Anderson Cancer Center, 1515 Holcombe Blvd, Houston, TX 77030, USA; ${ }^{3}$ Department of Biostatistics, The University of Texas MD Anderson Cancer Center, 1400 Pressler St, Houston, TX 77030, USA; ${ }^{4}$ Department of Imaging Physics, The University of Texas MD Anderson Cancer Center, 1400 Pressler St, Houston, TX 77030, USA; ${ }^{5}$ Department of Abdominal Imaging, The University of Texas MD Anderson Cancer Center, 1400 Pressler St, Houston, TX 77030, USA; *Author for correspondence: vkundra@mdanderson.org

BioTechniques 69: 37-45 (July 2020) 10.2144/btn-2020-0021

First draft submitted: 3 March 2020; Accepted for publication: 15 April 2020; Published online: 6 May 2020

\section{ABSTRACT}

Methods for imaging orthotopic prostate tumors within the prostate or small tumors with extension outside the prostate are needed to more closely model human prostate tumors, which are most commonly located within the gland or may extend just through the gland. By comparing MR sequences, we found that the T2-based Dixon 'water only' sequence best visualized tumors within the prostate of mouse models in both young and old mice and that tumor weight derived from this sequence correlated highly with ex vivo tumor weight $\left(r^{2}=0.98, p<0.001, n=12\right)$. This should aid tumor detection, margin delineation and evaluation of tumor burden to enable studies including, but not limited to, evaluating the natural history of the disease, the mechanisms of action and the efficacy of therapeutic interventions.

\section{METHOD SUMMARY}

The method utilizes a T2-based Dixon 'water only' sequence to image orthotopic prostate tumors. The sequence results in high signal of the prostate, making it the most prominent object in the image; intermediate signal of the tumor; and low signal in the surrounding fat and other structures except the easily recognizable bladder. This enables visualization and margin delineation of the orthotopic prostate tumor. Region of interest analysis can be performed to calculate tumor volume and derive tumor weight.

KEYWORDS:

Dixon • mouse model • MRI • orthotopic prostate cancer • prostate cancer

Prostate cancer is the most commonly diagnosed noncutaneous malignancy in men, and the fourth leading cause of cancer death affecting men overall [1]. Several animal models have been developed to better understand prostate cancer and its progression [17]. Among these, orthotopic prostate tumor models are preferred to subcutaneous models because they more closely mimic clinical scenarios and the microenvironment; however, such tumors are rarely visible or palpable. Noninvasive imaging methods for visualizing orthotopic prostate cancer are limited but such methods are needed and require sufficient sensitivity and resolution to detect, delineate and monitor the growth of tumors in the prostate.

In patients, tumors within the prostate are most commonly detected by magnetic resonance imaging (MRI), which allows for noninvasive functional assessment and visualization of in vivo anatomy with high soft tissue contrast. Compared with other imaging modalities, $\mathrm{MRI}$ enables excellent soft tissue contrast, spatial resolution, deep tissue evaluation and 3D image representation without requiring the administration of radioactive isotopes [8]. In animal models of certain tumors, MRI has proved to be a valuable tool to track tumor growth longitudinally in vivo, enabling assessment of the response to therapy of, for example, prostate cancer in bone [9-15].

With orthotopically implanted cells, prostate tumors can be difficult or impossible to palpate, particularly when the tumors are small. Without imaging, prostate tumor presence or tumor volume measurement is possible only at necropsy. Imaging would allow identification of animals with tumors and exclude those without, thus reducing the number of animals needed to assess the effect of an intervention. Commonly, T1- or non-fat-suppressed T2-weighted MRI, and less commonly diffusion-weighted imaging, has been used for detecting and monitoring large prostate tumors in mice, commonly without any significant adjacent normal prostate tissue [7,12,13,16-18].

However, methods for imaging prostate tumors within the prostate or small tumors with extension outside it are needed to more closely model human prostate tumors, which are most commonly within the gland or may extend just through the gland. This requires imaging that enables separation of the tumor from the prostate and from adjacent fat/tissues. Here we address this gap in knowledge.

As an alternative to traditionally used T1- and T2-weighted MRI sequences, newer Dixon-based sequences with 'water only' and 'fat only' weighting can be used where the degree of weighting is based on the weighting of the original sequence [19]. In our previous work evaluating different MR imaging sequences at both 4.7- and 7-T field strengths, we found that Dixon-T2-based sequences can 
significantly improve normal mouse prostate conspicuity, and noted that prostate gland volume and hydration status decrease whereas glandular hyperplasia increases in old versus young mice [20]. However, prostate cancer was not studied in these two settings, both of which are used in animal studies. The optimal MR sequences for imaging orthotopic prostate cancer are unknown. Such imaging is needed for tumor detection and margin delineation and for evaluating tumor burden, for example, to assess therapeutic efficacy. In the current study, we compared multiple MR sequences to determine which best delineates orthotopic prostate cancer.

\section{Materials \& methods}

\section{Cells}

PC3 human prostate cancer cells, which model human prostate adenocarcinoma and form tumors in nude mice, were obtained from American Type Culture Collection (ATCC, VA, USA) and were further authenticated by via microscopic morphology and DNA characterization by the Characterized Cell Line Core at the University of Texas MD Anderson Cancer Center. Mycoplasma testing was done with the MycoAlert PLUS mycoplasma detection kit (Lonza, SC, USA). The cells were grown in RPMI (Invitrogen, CA, USA) with 10\% heat inactivated fetal bovine serum (Hyclone Laboratories, UT, USA) containing $50 \mathrm{IU} / \mathrm{ml}$ penicillin, $50 \mu \mathrm{g} / \mathrm{ml}$ streptomycin, and $2 \mathrm{mM} \mathrm{L-glutamine}$ (Invitrogen) in a $5 \% \mathrm{CO}_{2}$ atmosphere. These were used for animal studies within ten passages.

\section{Animal experiments}

MRI of the prostate of young ( 2 months, $n=6)$ and old (18 months, $n=6)$ male nude mice was performed at 7 T. The animal experiments were approved by the institutional animal care and use committee and all experiments were performed in accordance with relevant guidelines and regulations. For the orthotopic xenograft mouse model, tumor was produced by implanting $50 \mu$ of cell suspension (50,000 PC3 cells) orthotopically in the exposed prostate gland through a surgical incision in the lower abdomen. Prior to incision, animals were anesthetized with $2 \%$ isoflurane and cleaned with betadine. Injections into the prostate were performed under microscope guidance using a microinjector [21]. MRI was performed at weeks 2, 4 and 5. Afterwards, all the mice were sacrificed and prostate tumors were confirmed and weighed.

\section{Magnetic resonance imaging protocol}

All mice were imaged 14 days after tumor implantation. Our previous work has demonstrated that the prostate is better visualized at 7 T than 4.7 T [20]. Thus MRI was performed using a 7-T MR system (Biospec, Bruker Biospin MRI, Inc., MA, USA). Transmit/receive volume coils with an inner diameter of $35 \mathrm{~mm}$ were used. Animals were anesthetized with $2 \%$ isoflurane for all MRI experiments, and phantoms (water or canola oil in PCR tubes) were imaged with the animal. Proper animal positioning was verified using a fast gradient echo localizer scan. The following seven different MRI sequences were used to acquire images in the axial plane with $4 \times 3 \mathrm{~cm}^{2} \mathrm{field}$ of view, $256 \times 192$ matrix, and $156 \mu \mathrm{m}$ spatial resolution unless otherwise noted:

- a T2-weighted Fast spin echo (FSE) acquisition (echo time 65 ms; echo train length 12; receiver bandwidth 101 kHz; repetition time $4000 \mathrm{~ms}$; nex 3; slice thickness $0.75 \mathrm{~mm}$ );

- a T2-weighted FSE with Dixon fat/water separation (echo time 60 ms; echo train length 8; repetition time 3700 ms; nex 2; slice thickness $0.5 \mathrm{~mm})$;

- a T2-weighted FSE acquisition with fat saturation (T2-FS) (echo time 65 ms; echo train length 12; receiver bandwidth 101 kHz; repetition time $4000 \mathrm{~ms}$; nex 3; slice thickness $0.75 \mathrm{~mm}$ );

- a FSE proton density-weighted sequence with Dixon fat/water separation (echo time $7 \mathrm{~ms}$; echo train length 8; repetition time $3000 \mathrm{~ms}$; nex 2; slice thickness $0.5 \mathrm{~mm}$ );

- a respiratory-gated diffusion-weighted imaging sequence (DWI) (echo time $33 \mathrm{~ms}$; B value $300 \mathrm{~s} / \mathrm{mm}^{2}$; repetition time $\sim 2 \mathrm{~s}$, determined by respiratory rate; nex 1 ; slice thickness $1 \mathrm{~mm}$; matrix $128 \times 128$; spatial resolution $312 \mu \mathrm{m})$.

In addition, we acquired a series of axial, dynamic-contrast-enhanced (DCE) images during and after 1:4 diluted gadopentetate dimeglumine contrast injection via a tail-vein catheter $(110 \mu, 0.25 \mathrm{M})$ using an interleaved fast spoiled gradient echo sequence (FSPGR) (TE/TR $1.672 \mathrm{~ms} / 50 \mathrm{~ms}$; in-plane resolution $312 \mathrm{~mm} \times 312 \mathrm{~mm}$; slice thickness $0.75 \mathrm{~mm}$; update rate $5.5 \mathrm{~s}$ per slice package).

\section{Prostate cancer measurements}

Prostate tumor volume measurements were performed using Image $\mathrm{J}$ software (NIH, MD, USA). The periphery of the prostate cancer on the MRI T2-based Dixon 'water only' images was manually traced on axial images using a region of interest (ROI), and the area of the enclosed region was calculated. Assuming a tumor density of $1 \mathrm{~g} / \mathrm{ml}$, tumor volumes $\left(\mathrm{mm}^{3}\right)$ were converted to weight ( $\mathrm{g}$ ) for analysis [22]. Contrast-to-noise ratio (CNR) of tumor to fat was calculated with measured signal intensities of the tumor and fat by manually placing a ROI on tumor and fat adjacent to the tumor in each slice of T2-FSE, T2-FSE-FS and T2-based Dixon 'water only' images, and dividing the difference in signal intensity of tumor to fat by the standard deviation in the reference water phantom. Similarly, CNR of prostate to tumor was calculated with measured signal intensities by placing a ROI on prostate and tumor in each slice of T2-FSE, T2-FSE-FS and T2-based Dixon 'water only' images and dividing the difference in signal intensity of prostate to tumor by the standard deviation in the reference water phantom [20]. 


\begin{tabular}{|c|c|c|}
\hline Group & Overall best sequence & p-value \\
\hline Young mice & T2-Dixon "water only" $>$ T2-FS = T2 $>$ FSPGR-DCE + contrast = DWI = PD-Dixon "water only" $>$ DCE without contrast & $<0.001$ \\
\hline Old mice & T2-Dixon "water only" $>$ T2-FS = T2 $>$ FSPGR-DCE + contrast = DWI = PD-Dixon "water only" $>$ DCE without contrast & $<0.001$ \\
\hline
\end{tabular}

Table 2. Comparison of prostate cancer conspicuity, margin delineation and tumor-to-background contrast in young and old nude mice at $7 \mathrm{~T}$.

$\begin{array}{llll}\text { Group } & \text { Sequence } & \text { Conspicuity } & \text { Border delineation } \\ \text { Young mice } & \text { T2-Dixon 'water only' vs all other } & \mathrm{p}<0.001 & \mathrm{p}<0.001 \\ \text { Old mice } & \text { T2-Dixon 'water only' vs all other } & \mathrm{p}<0.001 & \mathrm{p}<0.001\end{array}$

\section{Interpretation of MR images}

All MRI sequence images were arranged randomly and blinded interpretation was done independently by a board-certified radiologist with $>15$-years' experience in abdomen/pelvis imaging and a research scientist with $>12$-years' experience in small animal imaging to rank the best overall sequence for tumor visualization and to rank quality of prostate cancer conspicuity, margin delineation and tumorto-background contrast. The images of each mouse were ranked in relative gradations from best (\#1, i.e., the lowest number) to worst compared with each other. The best combination of sequences for tumor visualization was identified for each mouse imaging session by the two readers independently and the combination frequency tallied.

\section{Statistical analysis}

Prostate tumor volumes/weights and CNR were compared using two-sided $t$ tests. Regression analysis comparing MR-derived and ex vivo tumor volumes/weights was performed using Microsoft Excel 2007 (Microsoft Corp., WA, USA) and for all results, a two-tailed $p$ value of less than 0.05 was considered significant. Power calculation suggested that six samples per group would provide $\geq 80 \%$ power for $\alpha=0.05$ for primary end point overall best sequence. The power calculation used a repeated measures ANOVA design with each subject having seven different sequences and a Greenhouse-Geisser corrected $\mathrm{F}$ test with standard deviation for each sequence assumed to be 1.5 and correlation of 0.1 between two sequences.

For all the MR sequences blinded interpretation results, multiple comparison using the Tukey-Kramer method was used to compare the overall ranking, conspicuity, margin delineation and tumor-to-background contrast of tumors in young and old mice. The linear mixed model was used to estimate and compare ranking scores for sequences, adjusted for young/old mice. Differences between sequences were considered statistically significant if $\mathrm{p}<0.05$.

\section{Results \& discussion}

Visualization of orthotopic prostate tumor

Representative MR images demonstrate tumor in the prostate gland at 2 weeks (Figure 1A, Supplementary Figure 11A, Supplementary Figure $21 \mathrm{~A}$ ) and 4 weeks (Figure 1B; Supplementary Figure 11B, Supplementary Figure $21 \mathrm{~B}$ ) after orthotopic tumor inoculation in old (top) and young (bottom) mice. On the T2-based Dixon 'water only' sequences, fat is suppressed and the prostate appears as an area of high signal (bright), the primary object in the image. Tumor appears as an area of signal lower than the prostate tissue but higher than the adjacent suppressed fat. The T2-based Dixon 'water only' sequence ranked the highest for best overall tumor visualization as compared with the other MRI sequences for young and old nude mice (Table 1; $p<0.001, n=18, n=18$, Supplementary Data Table for Table 1). The T2-based Dixon 'water only' sequence also ranked the highest for overall tumor conspicuity, margin delineation and tumorto-background contrast as compared with other MRI sequences of young and old mice (Figure 2; $<<0.001, n=36$ ). Overall, T2-based Dixon 'water only' was superior to other MRI sequences for prostate cancer conspicuity, margin delineation and tumor-to-background contrast for young or old nude mice (Table 2; $\mathrm{p}<0.001, \mathrm{n}=18,18$, Supplementary Data Table for Table 2). Furthermore, no significant difference was seen in cancer conspicuity, border delineation or tumor-to-background contrast between young and old mice $(n=18$, Supplementary Table 3). T2-based Dixon 'water only' had greater tumor-to-fat CNR compared with T2 or T2 fat-suppressed sequences (Figure $3 A ; n=6, p<0.05$ ) for young or old nude mice. The prostate-to-tumor CNR of T2 Dixon 'water only' was similar to or less than T2 fat-suppressed or T2 images in young and old mice (Figure 3B).

\section{Prostate tumor volume/weight}

All tumors found at necropsy were well visualized in the T2-based Dixon 'water only' MR images. Prostate tumor volumes increased with time in both young and old mice (Figure 4A \& B). At 2 weeks, all mice had tumors visible by MRI in the prostate. Submillimeter tumors were identified. Figure 4C (Supplementary Figure 2 4C) demonstrates longitudinal Dixon 'water only' imaging of tumors in old (top) and young (bottom) mice, including 0.3-mm tumor in the former and 0.4-mm tumor in the latter at 2 weeks. By week 4, tumor excrescences, when present, tended to be located superior and anterior to the prostate. A highly statistically significant correlation was found between 


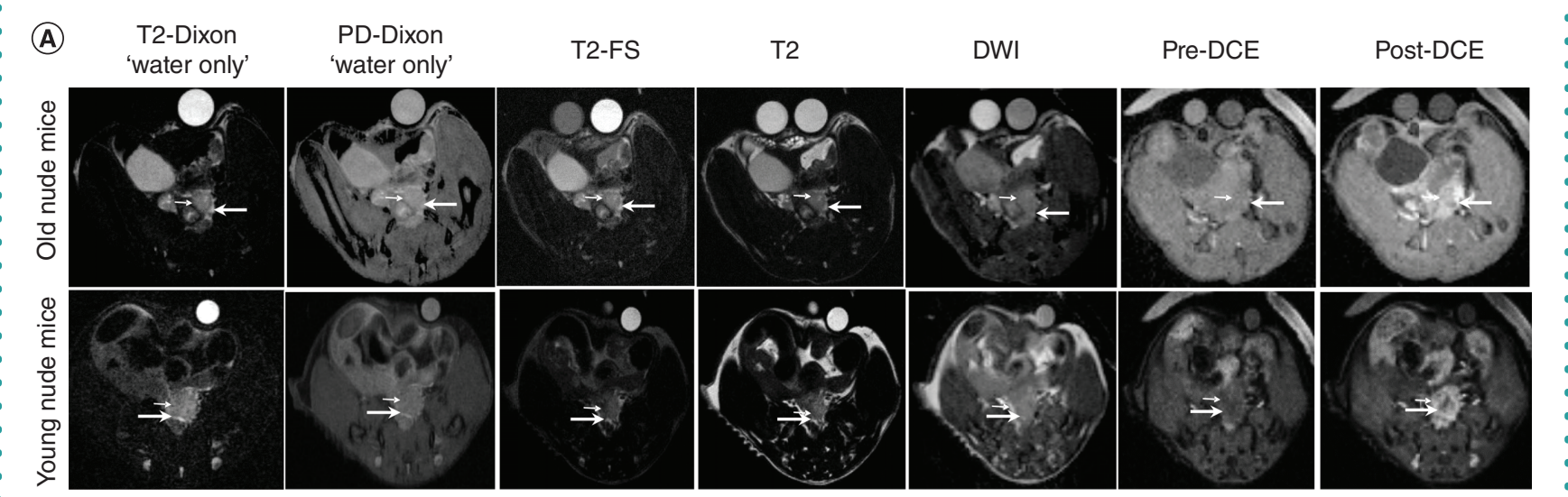

(B)
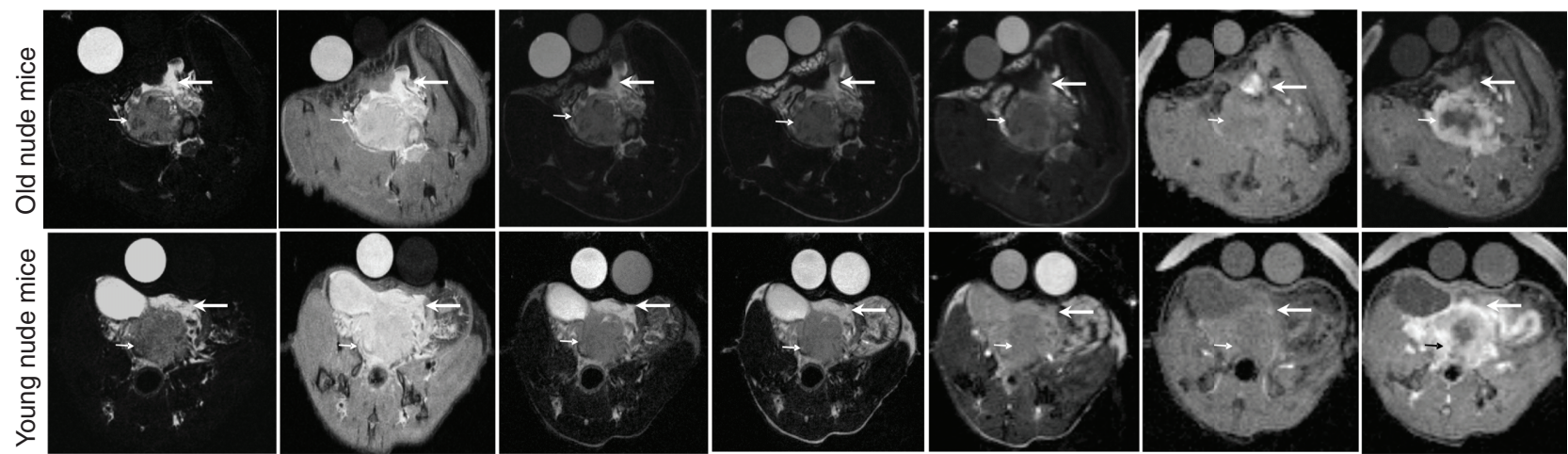

Figure 1. Representative magnetic resonance images of orthotopic prostate cancer in old (top) and young (bottom) nude mice. Multiple sequences were acquired at 7 T (A) 2 weeks and (B) 4 weeks after tumor inoculation. Circular objects anterior to the mouse are water phantoms (right on A and left on B; brighter on T2-Dixon 'water only') and canola oil phantoms (left on A and right on B) in PCR tubes.

Long arrow: Prostate; Short arrow: Tumor.

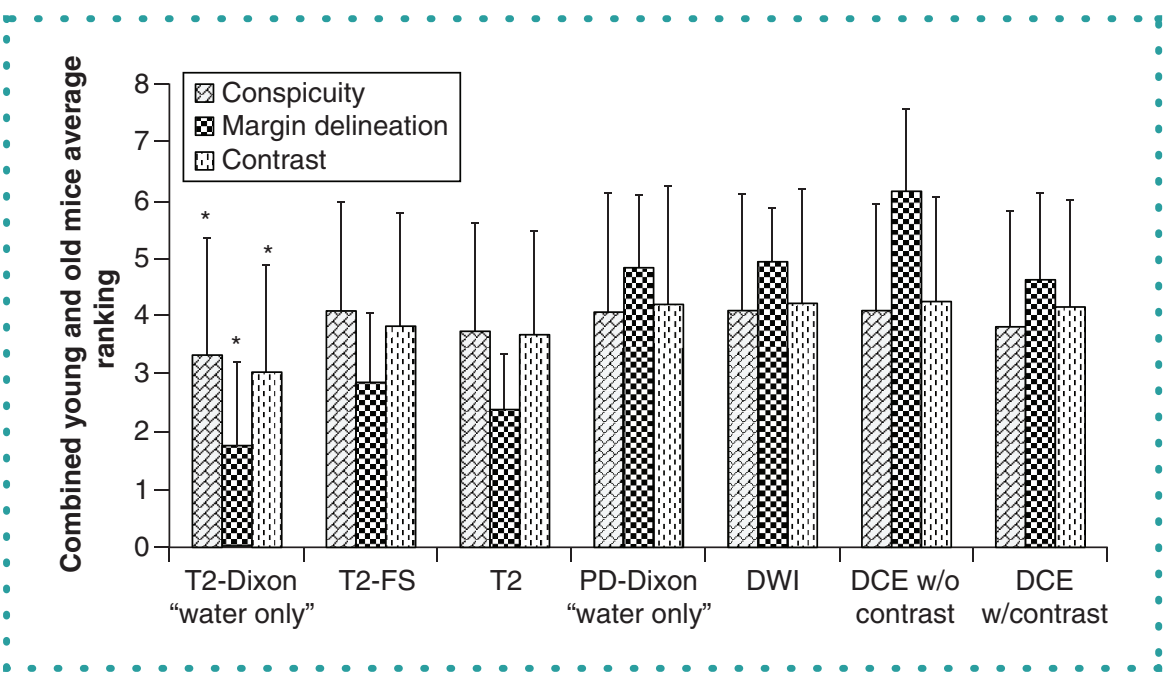

Figure 2. Overall ranking of mouse prostate cancer conspicuity, margin delineation and tumor-to-background contrast in both young and old nude mice. ( ${ }^{\star} \mathrm{p}<0.001, \mathrm{n}=36, \mathrm{~T} 2$-Dixon 'water only' vs all other sequences). 


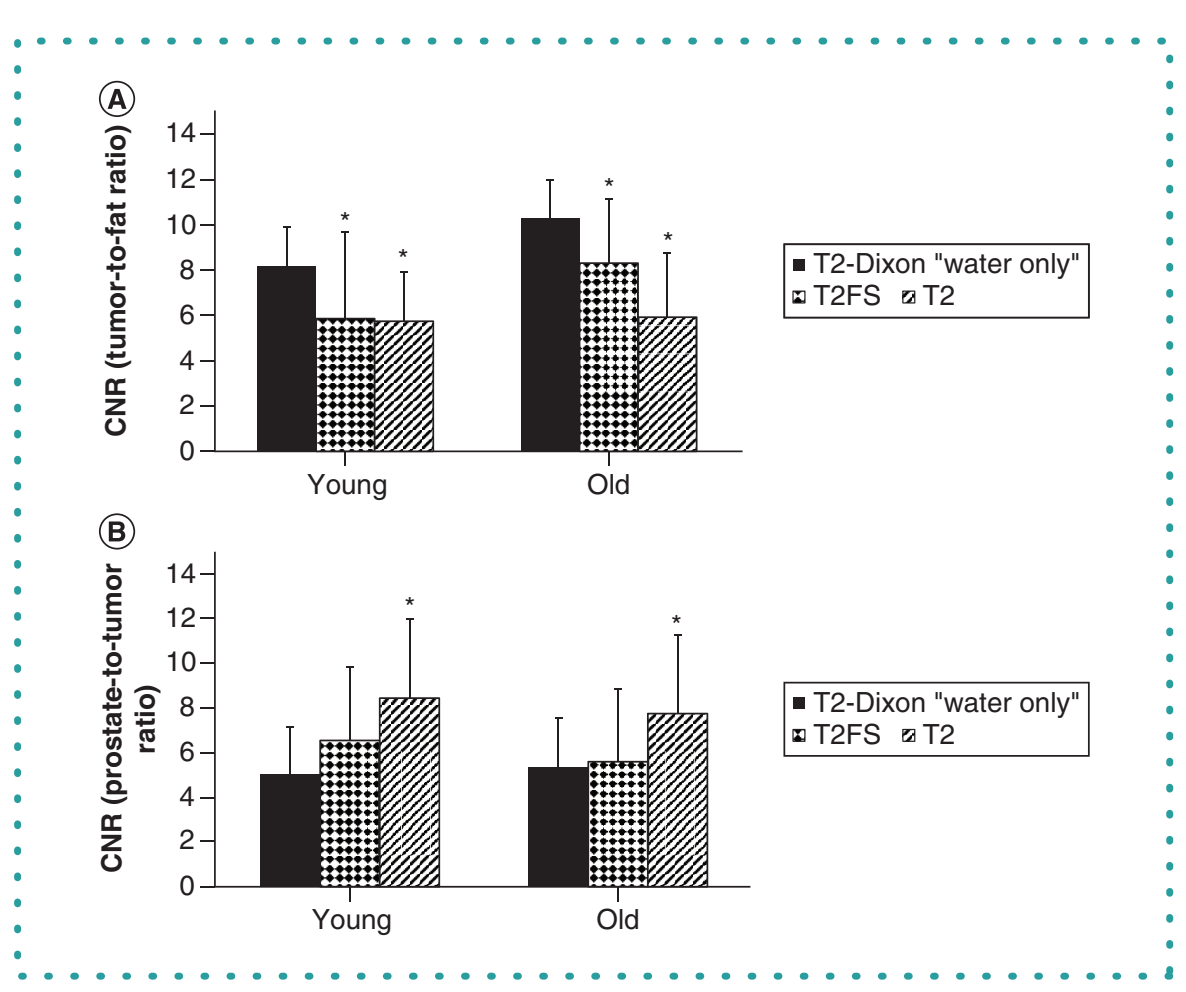

Figure 3. CNR measurements of tumor-to-fat and prostate-to-tumor. Contrast-to-noise ratio of tumor to fat (A) or prostate to tumor (B). Ratio measured using T2-Dixon 'water only', T2 and T2-FS sequences in young or old nude mice at 7 T ( ${ }^{\star} p<0.001, n=6$, compared with T2-Dixon water only for young or old mice respectively).

necropsy-determined tumor weights and the tumor weights derived from T2-Dixon 'water only' images $\left(r^{2}=0.98, n=12, p<0.001\right.$, coefficient of the $x$ variable $=1.15$; Figure $4 D$ ). Because multiple MR sequences are used for interpretation clinically, we further evaluated which combination of sequences best visualized the tumor in both young and old mice; T2-Dixon 'water only' was nearly always included in the combinations selected. Overall, the combination of sequences selected most frequently as the best was T2-Dixon 'water only' and T2 (Figure 5).

The T2-Dixon 'water only' sequence provided superior depiction of cancer within the prostate gland compared with the other sequences tested in the mouse models. Tumors were within the prostate or small tumors extending outside the prostate, modeling clinical prostate cancer, which is most commonly contained within the gland or may extend just through it. This required imaging that enabled distinction of the tumor from the prostate and from adjacent fat/tissues. Orthotopic tumor xenografts represent a practical model for preclinical studies of tumor development and response to therapy. Orthotopic implantation enables interaction of tumor cells with organ site-dependent microenvironmental factors which can modulate tumor growth patterns, progression and metastatic potential. Penet et al. found that human prostate tumor xenografts grown orthotopically had higher vascular volume, permeability, total choline and a more acidic extracellular $\mathrm{pH}$ in addition to more mitotic figures than those grown subcutaneously [23]. Although mouse subcutaneous tumor models are easy to establish and monitor, they often do not replicate biology as well as the original orthotopic anatomic site. However, orthotopic implantation also makes it more difficult to detect tumors and monitor their response to therapy over time.

MRI has not been optimized in mouse models of orthotopic prostate cancer. T1- or T2-weighted imaging have primarily been performed for large tumors where differentiation from the prostate and adjacent structures is not needed $[7,12,13,16-18]$. Clinically, a great majority of localized prostate cancers are within the gland; in later tumor stages, the bulk of the tumor is within the gland and has extensions outside the confines of the prostate. A prostate tumor larger than the prostate itself is very rare in patients. Clinically, MR is used for imaging prostate cancer within the prostate [24-27].

In mice, luciferase imaging has been espoused for tumor detection; however, the technique is limited because it requires gene transfer, which may alter the tumor cell and may not be feasible outside of cell lines, such as in tumor transplantation models like patientderived xenografts. Additionally, it most commonly results in $2 \mathrm{D}$ image representation, and can have significantly poorer correlation with tumor burden than MRI [28]. Moreover, due to poor depth of penetration, luciferase imaging is not translatable for percutaneous imaging of prostate cancer in humans. In contrast, MRI does not require gene transfer to image the tumor, and thus is applicable to cell lines, transplanted tumor tissue, and spontaneous prostate tumors such as those in transgenic models. MRI also enables 3D image representation and, as we found, results in very high correlation with tumor burden in the orthotopic prostate tumor model. 


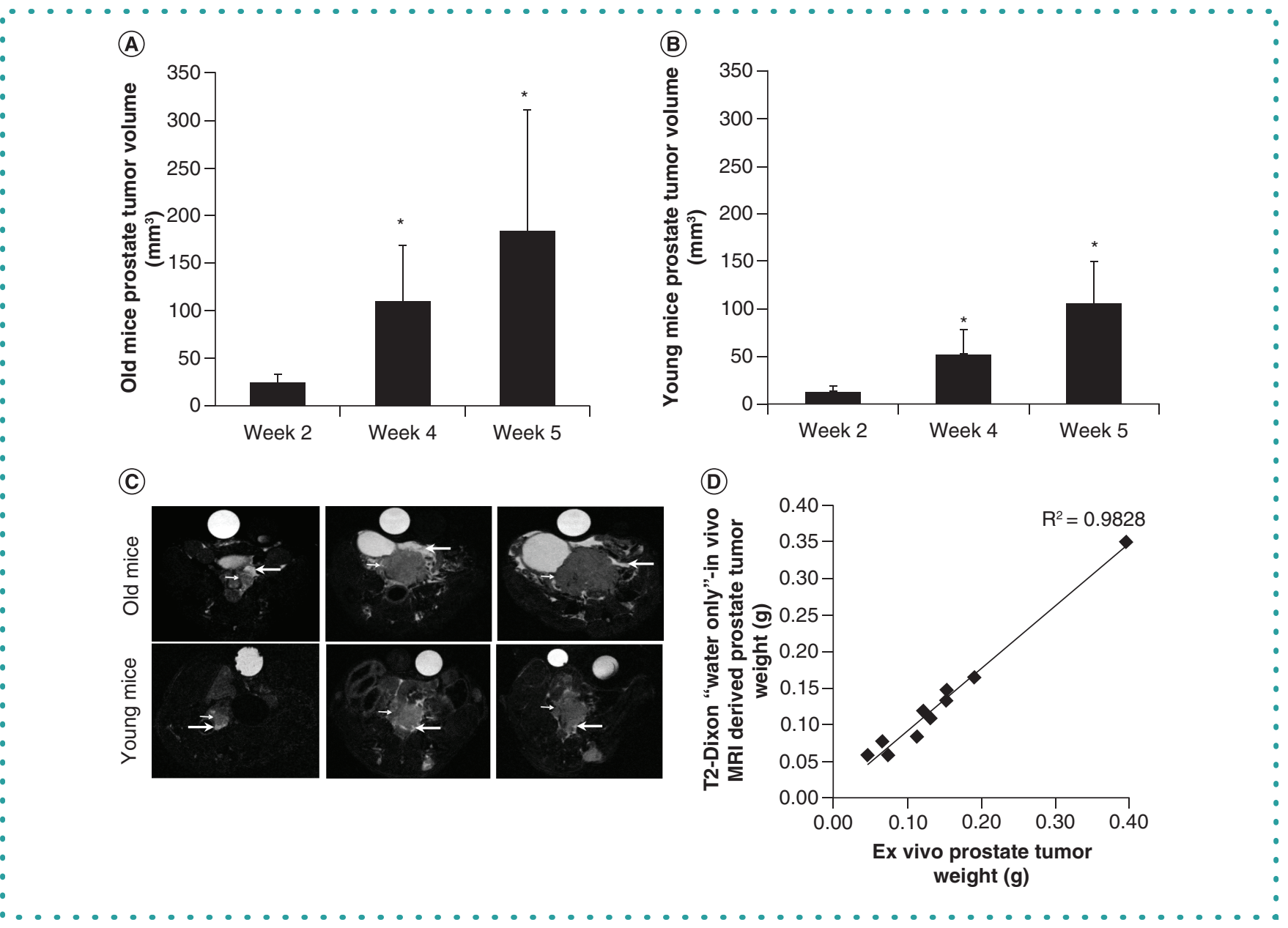

Figure 4. Tumor progression over time. Old (A) and young (B) nude mice prostate cancer volumes derived from week 2, 4, and $5 \mathrm{MR}$ images ( ${ }^{\star} \mathrm{p}<0.05$, $\mathrm{n}=6$, compared with week 2). (C) Representative longitudinal T2-Dixon 'water only' magnetic resonance images of orthotopic prostate cancer in old (top) and young (bottom) mice at 2 (left), 4 (middle), and 5 (right) weeks. (D) Correlation of tumor weight in young and old mice derived from T2-Dixon 'water only' sequence magnetic resonance images with tumor weight at necropsy at week $5\left(r^{2}=0.98, n=12\right)$.

Long arrow: Prostate; Short arrow: Tumor.

Selection of the optimal MRI sequence to use is essential during the acquisition of images. The current study evaluated the utility of several MR sequences to detect and longitudinally follow the prostate tumor noninvasively in a mouse model. A linear, highly correlated relationship between necropsy weights and T2-Dixon 'water only' MRI-derived tumor weights was found. We were able to detect submillimeter tumors within the prostate at week 2. Commonly, investigations that assess the efficacy of treatment strategies for prostate cancer use direct measurement of excised tumor weight/size as a measure of tumor progression following euthanasia. However, this method cannot follow orthotopic prostate tumors in the same animals longitudinally over the duration of treatment, as is done in patients. Such monitoring is, however, possible using the noninvasive in vivo imaging strategies described here. In our previous study, we showed that the T2-Dixon 'water only' MRI sequence is superior to other MR imaging sequences in visualizing the normal prostate gland of young and old mice [20]. MR signal changes in the mice associated with benign prostatic hyperplasia and change in prostate size with age were noted. We also found imaging at $7 \mathrm{~T}$ to be superior to imaging at $4.7 \mathrm{~T}$ for imaging the prostate gland [20]. Prostate tumors were not studied. Thus, in the current study, we compared multiple MRI sequences at $7 \mathrm{~T}$ for visualizing prostate cancer within the gland.

With the current 7 T MRI system, we were able to detect submillimeter size prostate cancer by in vivo imaging using a T2-based Dixon 'water only' sequence, which ranked the highest in overall image quality compared with the other sequences. T2-based Dixon 'water only' had greater tumor-to-fat CNR compared with T2 or T2 fat-suppressed sequences for young and old mice. The prostate-to-tumor CNR of T2-based Dixon 'water only' was similar to or less than T2 fat-suppressed or T2 sequences in young and old mice. Background can affect object perception; the superior dark background of the fat by Dixon 'water only' isolates the prostate and appears per CNR to have played a greater role in increasing tumor conspicuity [29]. The results support the use of the T2-Dixon 'water only' imaging sequence at $7 \mathrm{~T}$ for imaging orthotopic prostate cancer in young and old mice. The T2-Dixon 'water only' MRI-derived tumor weight demonstrated excellent correlation with prostate tumor weight at necropsy $\left(r^{2}=0.98\right)$, suggesting this method may be used to measure tumor size, 


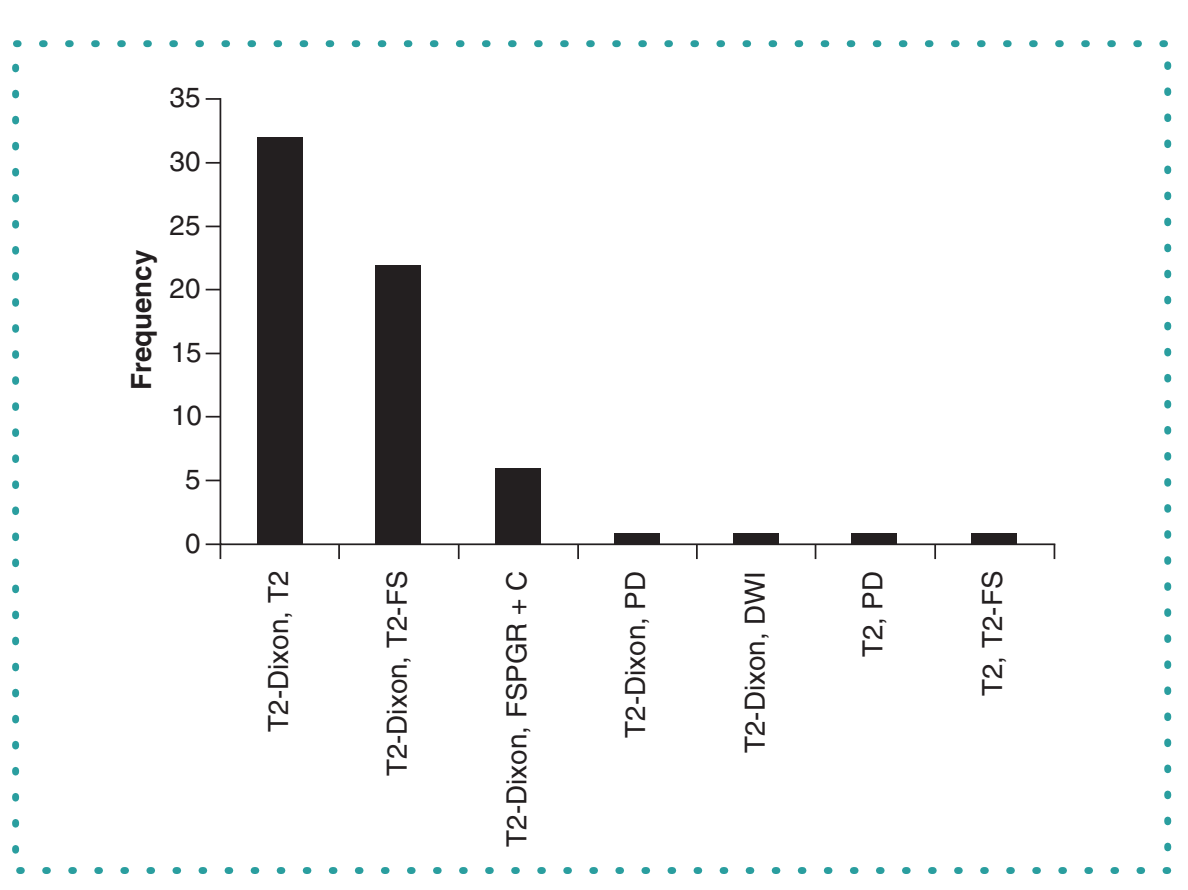

Figure 5. Comparison of best combination of sequences to visualize prostate tumor in mouse models.

for example to evaluate therapeutic efficacy. One limitation of this work is that we tested one cell line representative of human prostate cancer; however, similar results were seen in young and old mouse models and we predict applicability in a wide range of mouse models of orthotopic prostate cancer. Although we did not evaluate multiple cell lines, transgenic or PDX models, we expect our findings to be applicable to these models, because they should form more solid, less water-containing structures compared with the normal glandular structure of the prostate [20].

The prostate is a secretory gland and a much more hydrated structure compared with other organs in the male pelvis [20]. T2-Dixon 'water only' imaging takes advantage of this 'hydrated' state, resulting in a bright-appearing (high signal) prostate relative to surrounding structures. Prostate tumor appeared as relatively low signal in comparison; this enables distinction of prostate tumor from the prostate itself. The tissue surrounding the prostate is fat, which on a T2-FSE-type sequence appears relatively bright and can be difficult to distinguish from other structures, including tumors; fat suppression can aid differentiation. Dixon-based sequences can provide superior fat suppression compared with standard spectral fat suppression [30-32]. Fat-water swaps can occur with Dixon-based techniques, most commonly in separate limbs with air between them; however, we did not see this in our study.

Clinically, multiparametric MRI is used to visualize prostate cancer including T1, T2, DWI, and intravenous contrast-enhanced dynamic imaging (DCE) [24,25,33]. T2-weighted Dixon 'water only' imaging is not standard in this setting clinically. We used a small animal MRI as a cognate of clinical MRI systems and found that T2-weighted Dixon 'water only' was superior for visualizing tumor in the prostate compared with T2, T2-FS, DWI and DCE in the mouse model. Testing the utility of T2-based Dixon 'water only' in patients may be attempted in future studies. Clinically, combinations of sequences are used to find and characterize prostate tumors within the gland. We found that, of the tested combinations in the animal model for prostate tumor visualization, T2-based Dixon 'water only' was most frequently picked, most often with T2-FSE.

In summary, this study demonstrated that compared with other MR sequences tested, the T2-based Dixon 'water only' MRI sequence was superior for visualizing prostate tumors in mouse models mimicking clinical prostate cancer that is most commonly located within or extending just outside the prostate gland. The current study may prompt further studies for imaging signs of subtle and gross extraprostatic extension. Conspicuity, contrast and, in particular, margin delineation afforded by these methods should enable utilization by investigators.

\section{Future perspective}

The noninvasive MRI method enables serial imaging of mouse models for visualizing and characterizing the prostate and detecting and monitoring orthotopic tumors, enabling applications that may include investigating the pathogenesis of prostate cancer, understanding mechanisms of action and evaluating the efficacy of new therapies [20]. We expect that the techniques described here will be applicable to orthotopic, PDX and transgenic models, and that the field will be studying small tumors within or just outside the prostate, as found in humans, to better understand prostate cancer mechanistically and to create and test new therapies to combat the disease. 


\section{Author contributions}

M Ravoori performed the experiments and data analysis, wrote the draft of the paper and approved the final draft. S Singh performed the experiments, assisted with data analysis, helped drafting the paper and approved the final draft. P Yang supervised the cell inoculations for the orthotopic model, helped drafting the paper and approved the final draft. W Wei assisted with the statistical analysis, helped drafting the paper and approved the final draft. $\mathrm{H}$ Chen performed the cell inoculations and approved the final draft. J Ma assisted with the image acquisition (particularly of the Dixon images) and approved the final draft. $\mathrm{J}$ Bankson assisted with the image acquisition and interpretation and approved the final draft. $\mathrm{V}$ Kundra formulated the concept and design, helped perform the experiments and data analysis, drafted the paper and approved the final draft.

\section{Financial \& competing interests disclosure}

Funding was provided by the Prostate Cancer Research Program at The University of Texas MD Anderson Cancer Center and by the Cancer Center Support Grant P30 CA016672. The funders had no role in decisions on whether to publish the work. The authors have no other relevant affiliations or financial involvement with any organization or entity with a financial interest in or financial conflict with the subject matter or materials discussed in the manuscript apart from those disclosed.

No writing assistance was utilized in the production of this manuscript.

\section{Open access}

This work is licensed under the Attribution-NonCommercial-NoDerivatives 4.0 Unported License. To view a copy of this license, visit http://creativecommons.org/licenses/by-nc-nd/4.0/

\section{References}

Papers of special note have been highlighted as: $\bullet$ of interest; $\bullet \bullet$ of considerable interest

1. Talamini G, Zamboni G, Salvia R et al. Intraductal papillary mucinous neoplasms and chronic pancreatitis. Pancreatology 6(6), 626-634 (2006).

2. Greenberg NM, DeMayo F, Finegold MJ et al. Prostate cancer in a transgenic mouse. Proc. Natl Acad. Sci. USA 92(8), 3439-3443 (1995).

3. De Velasco MA, Tanaka M, Yamamoto Y et al. Androgen deprivation induces phenotypic plasticity and promotes resistance to molecular targeted therapy in a PTEN-deficient mouse model of prostate cancer. J. Carcinogenesis 35(9), 2142-2153 (2014)

4. Davies AH, Wang Y, Zoubeidi A. Patient-derived xenografts: a platform for accelerating translational research in prostate cancer. Mol. Cell. Endocrinol. 462(Pt A), 17-24 (2018).

5. Canesin G, Evans-Axelsson S, Hellsten R et al. Treatment with the WNT5A-mimicking peptide Foxy-5 effectively reduces the metastatic spread of WNT5A-low prostate cancer cells in an orthotopic mouse model. PLoS ONE 12(9), e0184418 (2017).

6. Rea D, Del Vecchio V, Palma G et al. Mouse models in prostate cancer translational research: from xenograft to PDX. Biomed. Res. Int. 2016, 9750795 (2016)

7. Valkenburg KC, Williams BO. Mouse models of prostate cancer. Prostate Cancer 2011, 895238 (2011).

8. Kurhanewicz J, Vigneron DB, Males RG, Swanson MG, Yu KK, Hricak H. The prostate: MR imaging and spectroscopy. Present and future. Radiol. Clin. North Am. 38(1), 115-138 viii-ix (2000).

9. Ravoori MK, Singh SP, Lee J, Bankson JA, Kundra V. In vivo assessment of ovarian tumor response to tyrosine kinase inhibitor pazopanib by using hyperpolarized ${ }^{13} \mathrm{C}$-pyruvate MR spectroscopy and ${ }^{18}$ F-FDG PET/CT imaging in a mouse model. Radiology 285(3), 830-838 (2017).

10. Bankson JA, Ji L, Ravoori M, Han L, Kundra V. Echo-planar imaging for MRI evaluation of intrathoracic tumors in murine models of lung cancer. J. Magn. Reson. Imaging 27, 57-62 (2008).

11. Ueda K, Kawashima H, Ohtani S et al. The 3p21.3 tumor suppressor NPRL2 plays an important role in cisplatin-induced resistance in human non-small-cell lung cancer cells. Cancer Res. 66(19), 9682-9690 (2006).

12. Eng MH, Charles LG, Ross BD et al. Early castration reduces prostatic carcinogenesis in transgenic mice. Urology 54(6), 1112-1119 (1999).

13. Kumar AP, Bhaskaran S, Ganapath M et al. Akt/cAMP-responsive element binding protein/cyclin D1 network: a novel target for prostate cancer inhibition in transgenic adenocarcinoma of mouse prostate model mediated by Nexrutine, a Phellodendron amurense bark extract. Clin. Cancer Res. 13(9), 2784-2794 (2007).

14. Kundra $\mathrm{V}, \mathrm{Ng} \mathrm{CS}, \mathrm{Ma} \mathrm{J}$ et al. In vivo imaging of prostate cancer involving bone in a mouse model. Prostate 67(1), 50-60 (2007).

15. Wan X, Corn PG, Yang J et al. Prostate cancer cell-stromal cell crosstalk via FGFR1 mediates antitumor activity of dovitinib in bone metastases. Sci. Transl. Med. 6(252), 252ra122 (2014).

16. Abdulkadir SA, Qu Z, Garabedian E et al. Impaired prostate tumorigenesis in Egr1-deficient mice. Nat. Med. 7, 101-107 (2001).

17. Mallett CL, Lim H, Thind K et al. Longitudinal anatomical and metabolic MRI characterization of orthotopic xenograft prostate tumors in nude mice. J. Magn. Reson. Imaging 40(4), 848-856 (2014).

18. Chen HY, Larson PEZ, Bok RA et al. Assessing prostate cancer aggressiveness with hyperpolarized dual-agent 3D dynamic imaging of metabolism and perfusion. Cancer Res. 77(12), 3207-3216 (2017)

19. Dixon WT. Simple proton spectroscopic imaging. Radiology 153(1), 189-194 (1984).

-. Describes the detailed Dixon methodology/technique that was used in the present study.

20. Ravoori M, Duggal J, Gagea M et al. Visualizing the prostate gland by MR imaging in young and old mice. PLoS ONE 8(3), e55746 (2013).

21. Bex A, Luboldt H, Sudermann T, Rembrink K, Otto T, Rubben H. Influence of linomide on local tumor growth and metastasis of the human hormone-resistant prostate cancer cell line PC3 in an orthotopic model. Eur. Urol. 37, 628-633 (2000).

- Describes the implantation technique for producing orthotopic prostate cancer.

22. Yang D, Han L, Kundra V. Exogenous gene expression in tumors: noninvasive quantification with functional and anatomic imaging in a mouse model. Radiology 235(3), 950-958 (2005).

23. Penet MF, Pathak AP, Raman V, Ballesteros P, Artemov D, Bhujwalla ZM. Noninvasive multiparametric imaging of metastasis-permissive microenvironments in a human prostate cancer xenograft. Cancer Res. 69(22), 8822-8829 (2009).

24. Tan CH, Hobbs BP, Wei W, Kundra V. Dynamic contrast-enhanced MRI for the detection of prostate cancer: meta-analysis. AJR Am. J. Roentgenol. 204(4), W439-448 (2015).

25. Tan CH, Wei W, Johnson V, Kundra V. Diffusion-weighted MRI in the detection of prostate cancer: meta-analysis. AJR Am. J. Roentgenol. 199(4), 822-829 (2012).

26. Vache T, Bratan F, Mege-Lechevallier F, Roche S, Rabilloud M, Rouviere 0 . Characterization of prostate lesions as benign or malignant at multiparametric MR imaging: comparison of three scoring systems in patients treated with radical prostatectomy. Radiology 272(2), 446-455 (2014).

27. Weinreb JC, Barentsz JO, Choyke PL et al. PI-RADS Prostate Imaging - Reporting and Data System: 2015, Version 2. Eur. Urol. 69(1), 16-40 (2016).

28. Ravoori MK, Margalit O, Singh S et al. Magnetic resonance imaging and bioluminescence imaging for evaluating tumor burden in orthotopic colon cancer. Sci. Rep. 9, 6100 (2019).

- Compares magnetic resonance imaging with bioluminescence imaging for measuring orthotopic colon tumors.

29. Kingdom FA. Lightness, brightness and transparency: a quarter century of new ideas, captivating demonstrations and unrelenting controversy. Vision Res. 51(7), 652-673 (2011).

30. Costelloe CM, Kundra V, Ma J et al. Fast Dixon whole-body MRI for detecting distant cancer metastasis: a preliminary clinical study. J. Magn. Reson. Imaging 35(2), 399-408 (2012).

31. Ma J, Costelloe CM, Madewell ME et al. Fast Dixon-based multisequence and multiplanar MRI for whole-body detection of cancer metastases. J. Magn. Reson. Imaging 29(5), 1154-1162 (2009). 
32. Le-Petross H, Kundra V, Szklaruk J, Wei W, Hortobagyi GN, Ma J. Fast three-dimensional dual echo Dixon technique improves fat suppression in breast MRI. J. Magn. Reson. Imaging 31(4), 889-894 (2010).

33. Barentsz JO, Weinreb JC, Verma S et al. Synopsis of the PI-RADS v2 guidelines for multiparametric prostate magnetic resonance imaging and recommendations for use. Eur. Urol. 69(1), 41-49 (2016). 
